UG-FT-101/99

hep-ph/9908299

August 1999

\title{
FOUR FERMION CONTACT TERMS IN CHARGED CURRENT PROCESSES AND LARGE EXTRA DIMENSIONS
}

\author{
FERNANDO CORNET 円 \\ Departamento de Física Teórica y del Cosmos, \\ Universidad de Granada, 18071 Granada, Spain \\ MONICA RELAÑO \& \\ Instituto de Astrofísica de Canarias \\ E-38200 La Laguna, Tenerife, Spain \\ and \\ JAVIER RICO 向 \\ Institut für Teilchenphysik \\ ETHZ, CH-8093 Zürich, Switzerland
}

\begin{abstract}
We study the bounds that can be obtained on four-fermion contact terms from the experimental data for $e^{+} p \rightarrow \bar{\nu} X$ obtained at HERA

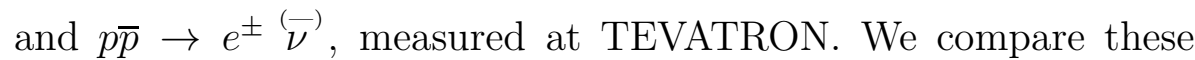
bounds with the ones available in the literature. Finally, we apply these results to study the compactification radius in theories with large extra dimensions and we obtain the bound $M_{c} \geq 3.3 \mathrm{TeV}$.
\end{abstract}

\footnotetext{
${ }^{1}$ E-mail address: cornet@ugr.es

${ }^{2}$ E-mail address: mpastor@ll.iac.es

${ }^{3}$ E-mail address: javier.rico@cern.ch
} 
The Standard Model is in excellent shape from the experimental point of view. The only problem appears to be the recently reported evidence for neutrino oscillations [1], but the agreement between the theoretical predictions and all the high energy experimental data is remarkably good [2]. Two years ago an excess of events in neutral $\left(e^{+} p \rightarrow e^{+} X\right)$ and charged current $\left(e^{+} p \rightarrow \nu_{e} X\right)$ Deep Inelastic Scattering was reported by the two HERA experiments: H1 [3] and ZEUS [4]. However, after an spectacular increase in the collected luminosity this possible hint for Physics Beyond the Standard Model has disappeared [5, 6]. It is now interesting (although certainly less exciting) to study the bounds that can be obtained from the new data on the mechanisms that were proposed as possible explanations for the excess of events.

The effects of new physics can be parametrized in terms of higher dimension $(d>4)$ operators [7]. In particular, the first operators contributing to Deep Inelastic Scattering are dimension 6, four-fermion contact interactions. These terms were introduced as an effective interaction relevant in case quarks and leptons were composite objects [8, 9]. However, it is clear that contact terms also appear as the low energy limit of the exchange of heavy particles, in the same way as the $W^{ \pm}$gauge boson exchange can be parametrized in the Fermi Lagrangian for energies much lower than $M_{W}$. The main difference between both approaches to contact terms is the interpretation of the mass scale $\Lambda$ they contain. In the first case, compositeness, the mass scale is related to the inverse of the size of the composite object. In the second case, $\Lambda$ is related to the mass and coupling constants of the exchanged particle.

A particularly interesting application of contact terms appears as a result of recent advances in superstring theories, where it has been observed that compact dimensions of a radius of $O\left(1 \mathrm{TeV}^{-1}\right)$ can be at the origin of supersymmetry breaking [10]. Also, even larger compact dimensions, with sub-millimeter compactification radius, allow to reduce the Planck scale to 
become of the order of a few $\mathrm{TeV}$, avoiding the gauge hierarchy problem [11]. So, if we have $6-n$ dimensions of $O\left(\mathrm{TeV}^{-1}\right)$ and $n \geq 2$ dimensions with a compactification size $O(m m-f m)$, in Type I/I' and Type IIA superstring theories we can achieve that the scale at which gravity becomes strong and the string scale are, both, $O(\mathrm{TeV})$ [12]. Gravitons propagate in the $n$-dimensional space, giving rise to new, effective interactions among the ordinary particles that live in our conventional four dimensional space-time [13]. Gauge bosons propagate in the $10-n$ dimensional space and in the ordinary four dimensional space appear as a Kaluza-Klein tower of states. Neutral current processes receive two types of new contributions: graviton exchange and KK-states exchange, while charged current processes only receive the second type of contributions. The phenomenology of these models is being discussed extensively in these days [14].

In this note we are going to use recent data from HERA $\left(e^{+} p \rightarrow \bar{\nu}_{e} X\right)$ and TEVATRON $\left(p \bar{p} \rightarrow e \bar{\nu}_{e} X\right)$ to obtain bounds on the mass scale appearing in the $e \nu q q^{\prime}$ contact term and we will compare them with the bounds obtained from the unitarity of the CKM matrix [15]. We will finish with a discussion of the connection of our results with extra dimensions physics.

Low energy effects of physics beyond the SM, characterized by a mass scale $\Lambda$ much larger than the Fermi scale, can be studied by a nonrenormalizable effective lagrangian, in which all the operators are organized according to their dimensionality. Since the energies and momenta that can be reached in present experiments are much lower than $\Lambda$, it is expected that the lowest dimension operators provide the dominant corrections to the SM predictions. Requiring $S U(2) \times U(1)$ invariance, the relevant lagrangian for $e \nu q q^{\prime}$ charged current processes including dimension 6 four-fermion operators is:

$$
\mathcal{L}=\mathcal{L}_{S M}+\eta^{l q}\left(\bar{l} \gamma_{\mu} \tau^{I} l\right)\left(\bar{q} \gamma^{\mu} \tau^{I} q\right)+\mathcal{L}_{S},
$$

where $\mathcal{L}_{S M}$ is the SM lagrangian, $l=(\nu, e)$ and $q=(u, d)$ are the $S U(2)$ doublets containing the left-handed lepton and quark fields, $\tau^{I}$ are the Pauli 
matrices and $\mathcal{L}_{S}$ are four fermion terms containing scalar currents instead of the vector currents shown explicitly in Eq. (1). It is customary to replace the coefficient $\eta$ by a mass scale $\Lambda$ :

$$
\eta=\frac{\epsilon g^{2}}{\Lambda^{2}}
$$

with $\epsilon= \pm 1$ taking into account the two possible interference patterns. For historical reasons $\Lambda$ is usually interpreted as the mass scale for new physics in the strong coupling regime, i.e. with

$$
\frac{g^{2}}{4 \pi}=1
$$

If the contact term is due to the $s$ or $t$ channel exchange of a heavy particle with a mass $M_{h}$ much larger than the center of mass energy then

$$
\eta=\frac{\bar{g}^{2}}{M_{h}^{2}} \text { and } \Lambda=\frac{\sqrt{4 \pi} M_{h}}{\bar{g}}
$$

where $\bar{g}$ is the coupling constant of the heavy particle to a fermion pair.

We will not consider the terms in $\mathcal{L}_{S}$ in our analysis because it has been shown that the ratio

$$
R=\frac{\Gamma\left(\pi^{ \pm} \rightarrow e^{ \pm} \nu\right)}{\Gamma\left(\pi^{ \pm} \rightarrow \mu^{ \pm} \nu\right)}=(1.230 \pm 0.004) \times 10^{-4}
$$

provides a very strong bound on these terms:

$$
\Lambda_{s}>500 \mathrm{TeV}
$$

This is due to the fact that the scalar currents appearing in $\mathcal{L}_{S}$ do not lead to helicity suppression in the pion decay amplitude as $V-A$ currents do [16, 17].

The expression for the cross-section $\sigma\left(e^{+} p \rightarrow \bar{\nu} X\right)$ can be found in Ref. [18], where a first analysis to the HERA data was presented. Here 
we perform a fit to the combined data for $d \sigma / d x d Q^{2}$ shown by the two experiments, H1 and ZEUS [5, 6], at the Moriond and Vancouver conferences. The total integrated luminosity collected is $37 p b^{-1}$ for $\mathrm{H} 1$ and $47 p b^{-1}$ for ZEUS. We have used the MRSA parameterization [19], but one cannot expect important changes in the result when using another parameterization. In our fit we have included Standard Model radiative corrections, but we have neglected the interference between these corrections and the new terms. Certainly, the data are compatible with the Standard Model predictions and we can only obtain $95 \%$ C.L. bounds on $\Lambda_{+}^{l q}$ and $\Lambda_{-}^{l q}$, where the subscript refers to the value of $\epsilon$. In order to obtain these bounds we have assumed that the probability density has the form:

$$
f(z, n)=\frac{z^{n / 2-1} e^{-z / 2}}{2^{n / 2} \Gamma(n / 2)},
$$

where $n=m-1$, and $m$ is the number of data points included in the fit. This expression corresponds to a $\chi^{2}$ distribution with $n$ degrees of freedom. The $95 \%$ C.L. bounds we have obtained are:

$$
\Lambda_{+}^{l q} \geq 3.5 \mathrm{TeV} \text { and } \Lambda_{-}^{l q} \geq 3.1 \mathrm{TeV}
$$

It is interesting to note at this point that, due to the dominance of the first family quarks in the proton structure functions, the results shown in Eq. (8) are strongly dominated by contact terms involving only first family quarks and leptons, i.e. an evud contact term. Indeed, neglecting new terms involving quarks from the second family we arrive to very similar bounds:

$$
\Lambda_{+}^{l q} \geq 3.2 \mathrm{TeV} \text { and } \Lambda_{-}^{l q} \geq 2.8 \mathrm{TeV}
$$

We now turn our attention to the closely related processes $p \bar{p} \rightarrow e^{ \pm}(\bar{\nu})$ measured at TEVATRON. At the partonic level this process is related to the one at HERA via $t$ to $s$ channel exchange. This, however, introduces a problem because the $W^{ \pm}$gauge boson can now be produced on-mass shell, 
producing a very large background to study new physics. In our fit we have used the data for $\frac{d \sigma}{d m_{t}}$, where $m_{t}$ is the $e \nu$ transverse mass, from Ref. 20] with $m_{t} \geq 110 \mathrm{GeV}$. This value has been chosen to optimize the sensitivity to new physics. The values for the $W$ mass and width we have used are: $M_{W}=(80.41 \pm 0.10) \mathrm{GeV}$ and $\Gamma_{W}=(2.06 \pm 0.06) \mathrm{GeV}$ 21] and we have checked that our results are not sensitive to changes of these parameters within one standard deviation. The bounds we obtain,

$$
\Lambda_{+}^{l q} \geq 2.0 \mathrm{TeV} \text { and } \Lambda_{-}^{l q} \geq 1.2 \mathrm{TeV},
$$

are much less restrictive than the ones obtained at HERA.

The most stringent bounds on lepton-quark charged current contact terms have been obtained from the observed unitarity of the CabbibboKobayashi-Maskawa matrix elements in Ref. [22]:

$$
\left|V_{u d}\right|^{2}+\left|V_{u s}\right|^{2}+\left|V_{u b}\right|^{2}=0.9965 \pm 0.0021
$$

Since CKM matrix elements are experimentally determined from the ratio of semileptonic to leptonic processes, both, lepton-quark and purely leptonic contact terms contribute to Eq. (11):

$$
V_{u d_{j}}^{o b s}=V_{u d_{j}}^{S M}\left(1-\frac{\eta^{l q}-\eta^{l l}}{8 \sqrt{2} G_{F}}\right),
$$

where $\eta^{l q}$ and $\eta^{l l}$ stand for the lepton-quark and purely leptonic, respectively, contact term couplings. The bounds on $\eta^{l q}$ and, consequently, on $\Lambda^{l q}$ depend on the ones that can be obtained for $\eta^{l l}$ (or $\Lambda^{l l}$ ). Hagiwara and Matsumoto have performed a fit to electroweak parameters measured at LEP1, TEVATRON and LEP2 to obtain a value for the $T$ parameter from which the bounds 22]:

$$
\Lambda_{+}^{l l} \geq 7.5 \mathrm{TeV} \quad \text { and } \quad \Lambda_{-}^{l l} \geq 10.2 \mathrm{TeV}
$$

were obtained. Introducing this result into Eqs. (11), (12) and assuming that the contact terms are the same for all three families they obtained:

$$
\Lambda_{+}^{l q} \geq 5.8 \mathrm{TeV} \text { and } \Lambda_{-}^{l q} \geq 10.1 \mathrm{TeV} .
$$


These bounds are more stringent than the ones obtained from HERA. However, one should notice that both sets of bounds are complementary because of the different assumptions used. Indeed, the result (14) rely on the assumption that $V_{u d}, V_{u s}$ and $V_{u b}$ receive the same contribution from contact terms, while the bounds obtained from ZEUS and $\mathrm{H} 1$ data are independent from this assumption, as we have explicitly shown in Eqs. (8) and (9). We should also point out that the HERA data we used in our fit has been obtained with positron beams. Since positrons interact via charged current processes with $d$ and $\bar{u}$ quarks in the proton, while electrons interact with $u$ and $\bar{d}$ quarks, the cross section with positrons in the initial state is much smaller at large $x$ and $Q^{2}$ than the one with electrons. Thus, with the same integrated luminosity using electron beams as the one collected up to now with positron beams the bounds shown in Eqs. (8) and (9) will improve in such a way that for $\Lambda_{+}^{l q}$ can become similar to the one in Eq. (14).

The contact terms we have been studying up to now can be easily related to the exchange of a tower of KK states corresponding to the $W$ boson. Such a tower appears when the number of space-time dimensions is larger than 4 and gauge bosons can propagate in the new dimensions. For energies lower than the inverse of the compactification radius $\left(R \sim 1 / M_{c}\right)$ the gauge bosons propagating in the new dimensions appear as a tower of states with the same couplings as the standard bosons. The lightest of the new states has a mass $O\left(M_{c}\right)$. For $M_{c} \geq O(1 \mathrm{TeV})$ it is, thus, justified to approximate the effects of the exchange of the new particles in four fermion processes by a contact term of the type introduced in Eq. (11).

The relation between the mass scale $\Lambda$ and the compactification scale $M_{c}$ is particularly simple in the case of only one extra dimension with compactification scale $O(\mathrm{TeV})$ :

$$
M_{c}^{2}=\frac{g^{2} \Lambda_{-}^{2}}{2 \pi} \sum_{n=1}^{\infty} \frac{1}{n^{2}},
$$


where $g$ is the $S U(2)$ coupling constant and the sum covers the contribution of the infinite number of states. This sum s finite and turns out to be $\pi^{2} / 6$. In case there are more than one extra dimension with the same compactification radius, the sum is divergent and a new parameter, a cut-off, must be introduced. Since the coupling of the new states to left handed fermions is universal, as it is the case for the $W$ boson, we just have take the most stringent bound for $\Lambda_{-}$and convert it into a bound for $M_{c}$. Thus, using $\Lambda_{-} \geq 10.2 \mathrm{TeV}$ we obtain

$$
M_{c} \geq 3.3 \mathrm{TeV}
$$

In summary, we have obtained bounds on the mass scale of the $S U(2) \times$ $U(1)$ invariant, four-fermion, charged current contact term from the recent HERA and TEVATRON data. The first ones appear to be more sensitive to the presence of this contact term, but more luminosity (especially with electron beams) is needed before the bounds obtained from these processes can be competitive with the ones obtained from the unitarity of the CKM matrix. Finally, we have converted these results into a bound on the compactification scale of a large extra dimension in which the $W$ boson can propagate, obtaining $M_{c} \geq 3.3 \mathrm{TeV}$. This result is particularly interesting, not only because it is one of the largest lower bounds obtained, but also because, being obtained form charged current processes, it is free from any assumption on the effects of the KK tower of the graviton in theories with a low gravity scale.

We thank A. Kotwal, K. Hagiwara, W. Hollik and M. Masip for very helpful discussions and comments. This research was partially supported by CICYT, under contract number AEN96-1672, and Junta de Andalucia, under contract FQM 101. 


\section{References}

[1] Super-Kamiokande Coll., Phys. Rev. Lett. 81 (1998) 1562; hepex/9807003.

[2] W. Hollik, Plenary talk at the XXIX Int. Conf. on High Energy Physics, Vancouver (Canada), July 1998. Preprint CERN-TH/98-358, KA-TP18-1998, hep-ph/9811313.

[3] H1 Coll., Z. Phys. C74 (1997) 191.

[4] ZEUS Coll., Z. Phys. C74 (1997) 207.

[5] H1 Coll., Talk at the XXIX Int. Conf. on High Energy Physics, Vancouver, July 1998, Abstract 751.

ZEUS Coll., Talk at the XXIX Int. Conf. on High Energy Physics, Vancouver, July 1998, Abstract 533.

[6] V. Chekelian, talk at the XXXIV Rencontres de Moriond, March 1999.

[7] W. Buchmüller and D. Wyler, Nucl Phys. B268 (1986) 621.

[8] M. Peskin and T. Takeuchi, Phys. Rev Lett. 65 (1990) 964.

[9] R. Rückl, Phys. Lett B129 (1983) 363; Nucl. Phys. B234 (1964) 91.

[10] I. Antoniadis, Phys. Lett. B246 (1990) 377.

A. Pomarol amd M. Quirós, Phys. Lett B438 (1998) 255, hepph/9806263.

I. Antoniadis, S. Dimopoulos, A. Pomarol and M. Quirós, Nucl. Phys. B544 (1999) 503, hep-ph/9810410.

A. Delgado, A. Pomarol and M. Quirós, hep-ph/9812489.

[11] N. Arkani-Hamed, S. Dimopoulos and G. Dvali, Phys. Lett. B429 (1998) 263; hep-ph/9803315 
[12] I. Antoniadis and B. Pioline, Large Dimensions and String Physics at a TEV, Talk presented at "Fundamental Interactions: from symmetries to black holes", Brussels (March 1999) and "Beyond the Desert 99", Castle Ringberg (Germany, June 1999), hep-ph/9906480.

[13] G.F. Giudice, R. Ratazzzi and J.D. Wells, Nucl. Phys. B544 (1999) 3, hep-ph/9811291.

T. Han, J.D. Lykken and R.J. Zhang, Phys. Rev. D59 (1999) 105006, hep-ph/9811350.

J.L. Hewett, Phys. Rev. Lett. 82 (1999) 4765, hep-ph/9811356.

E. Mirabelli, M. Perelstein and M.E. Peskin, Phys. Rev. Lett. 82 (1999) 2236, hep-ph/9811337.

[14] T.G. Rizzo, Phys. Rev. D59 (1999) 115010, hep-ph/9901209.

C.D. Carone, Phys. Lett. B454 (1999) 70, hep-ph/9902407.

M. Masip and A. Pomarol, hep-ph/9902467.

T.G. Rizzo and J.D. Wells, hep-ph/9906234.

W.J. Marciano, hep-ph/9903451.

P.H. Frampton and A. Rasin, hep-ph/9903479.

C.D. Carone, hep-ph/9907362.

[15] K. Hagiwara and S. Matsumoto, Phys. Lett. B424 (1998) 362; hepph/9712260

[16] G. Altarelli, G.F. Giudice and M.L. Mangano; Nucl. Phys. B506 (1997) $29 ;$ hep-ph/9705287.

[17] O. Shanker; Nucl. Phys. B204 (1982) 375.

[18] F. Cornet and J. Rico; Phys. Lett. B412 (1997) 343; hep-ph/9707299

[19] A.D. Martin, R.G. Roberts, W.J. Stirling, Phys. Rev. D50 (1994) 6734.

[20] C. Gerber; Review of $W$ and $Z$ Production at the TEVATRON; hepex/9806005. 
[21] Particle Data Group; The Eur. Phys. J. C3 (1998) 1.

[22] K. Hagiwara and S. Matsumoto; Phys. Lett. B424 (1998) 362; hep$\mathrm{ph} / 9712260$. 